\title{
Intellectualization of Interactive Electronic Technical Manuals Using Expert Systems with the Accumulation of Knowledge
}

\author{
A.V. Proletarsky ${ }^{1}$, A.A Gorin ${ }^{2}$, A.A. Wnukov ${ }^{1,2}$ \\ ${ }^{1}$ Department of Computer Science and Control Systems, Bauman Moscow State Technical \\ University, Moscow, 105005, Russia \\ ${ }^{2}$ National Research University Higher School of Economics, Bolshoy Trekhsvyatitelskiy Lane, 3, \\ Moscow, 109028, Russia
}

Keywords: expert system, interactive electronic technical manuals, synthesis, block diagram, database.

\begin{abstract}
This article describes the possibility of using expert systems (ES) in the framework of interactive electronic technical manuals. Invited to consider the benefits of diagnosis and prognosis of the technical condition of the product for the end user, also evaluate the capabilities of intellectualization accumulation of new declarative and procedural knowledge by experts. This can really enhance the intellectual component of the maintenance of modern and advanced equipment. The comparative analysis of the possibilities ES allowed to formulate requirements to clarify the criteria user requests. The article shows a block diagram of synthesis ES and IETM and its description. Named and described the types of knowledge of the expert system. The process of filling expert systems knowledge is automated.
\end{abstract}

\section{Introduction}

Currently in Russia exist IETM system supporting the entire lifecycle of products, which are developed with this product at all stages of production. However, such systems were made only for needs of the enterprises in which they were introduced and solve specific tasks automation development of electronic documentation. Basically interactive electronic technical manuals are finished products, which are developed with the device, which it describes. Organizations create IETM during production or after production of products from operational documentation. Paper documents can be converted into electronic form, then structuring it. The finished electronic product documentation is structured in accordance with the standards. Currently, there is some experience in creating e-operational documentation for complex military and civilian systems, such as the Su-35, Mi-8 and many others. In this case, IETM created during the production of the product could be used in the production process and maintenance equipment with distributed information management system and the general computerization of the equipment, but not without drawbacks.

The new formulation of the problem involves the implementation of an expert system synthesis and interactive technical manuals and create an innovative structure of the system, which allows to accumulate knowledge in all phases of the product lifecycle. And the appearance of specific product information in such a system should be presented in the form of a mathematical model used for diagnostics and prediction of its technical condition during operation.

The relevance of such a system would consist in the fact that it represents the intellectualization of interactive electronic technical manuals, provides users the necessary information about the product, which helps in the development of operational skills and technical support of interested product, facilitates the search for specific elements with the possibility of accumulation knowledge of experts.

The aim of this research is to formulate technical project for an experimental program complex of diagnostics and forecasting products, based on a proposal to develop a new structure and a new mechanism for the creation of IETM.

The practical creation of electronic technical manuals are reflected in the scientific literature. In steps being introduced - maintenance, operation and maintenance of complex products are often 
used automated monitoring systems and diagnostics. Data in systems are used together with information in the documentation. The approach is to use the technical information contained in the documentation in the form of a plurality of data blocks with attributive (ID-status-) and the content part. [1] describe the modern experience of research and practical work on the implementation of information technologies in industries with information support product lifecycle from design to utilization stage after the operation. System TG Builder, used for automated preparation of documentation in electronic and paper form for complex products is also described in [1]. [2] deals with basic approaches and methods of the design of expert systems technology, basic stages of the operation and the creation of systems, knowledge representation methods, algorithms and mathematical apparatus inference of knowledge, different approaches to the representation of uncertainty in expert systems.

\section{The effectiveness of the use of IETM with expert system}

Currently created IETM serve for the production and maintenance of products family of standard products for different purposes. The manuals are available instructions for maintenance and operation of a particular type or family of modern technology. Users should prompt the use of tools search for the desired item by its code or location of its own in a nested list, through the mechanism of opening of a significant number of tabs. This does not guarantee that the user will find the information he needs at the opening of a tab with the search element. Perhaps element herein would not repair information, as these parts are located in the neighboring section. May also be affairs with the operation of the product, as supported by the efficiency and performance of repair. In order not to fall into the described situation requires the creation of a model of the lifecycle support system that combines into one IETM and ES. The integration of these two systems into one allows you to use ES not only in advice mode and support for the formal description of a particular element of the system, but will also provide online help to the employee in solving search problems.

With ES user queries will give IETM. In turn, the integrated system, analyzing queries and giving on them answers and meaningful information, will itself lead poll worker clarifying its queries using single or multiple criteria, adding to own knowledge base. Adding intelligent features into an integrated system IETM and ES allow receiving answers to questions about the items and entering them into components and devices, which greatly facilitate the work of the staff.

When combined IETM and ES into one integrated system will need to create a knowledge base with sections, the relevant sections of IETM. The elements of a knowledge base refer to elements guide called data modules. All data modules have a unique number that is recognized by ES. Intelligent output machine by analyzing the specific needs of staff, will provide information on the set queries.

The choice of species of expert system is determined by the requirements to its primary and secondary support functions. An integrated system of IETM and ES should function in a dialogue with the staff in dealing with the operation of the product and assist in the repair of troubleshooting in order to then propose different solutions to the problem.

To solve the problem of estimating the effectiveness of IETM, built with the use of an expert system is required to formulate a mathematical formulation and an analytical study of the problem of formation of informed IETM containing operational documentation as to maximize the quality of IETM.

Thus, to evaluate the effectiveness of using IETM with ES is necessary to determine:

- the problem as an optimization with the objective function and constraints;

- type of target integrated indicator of quality IETM OD with ES;

- method of solving the problem by finding the maximum integral indicator of quality IETM by methods of numerical optimization;

- business process models of technology creation, maintenance and operation of IETM for development and production of operational documentation on the family of standard products of wide purpose. 
When defining the quality of IETM and ES must be defined the function and weighting coefficients also actually parameters on which it depends. As defined by measurable indicators that affect the quality of the IETM and ES, we can offer the following: completeness, accuracy, timeliness, costs of production, storage, use and maintenance of the product documentation with a certain quality. Thus it is possible to refer to the national experience and examples of systems with IETM and ES indicators of completeness - the number of CBMs in the EED 2450 - 3344, accuracy - the number of base classes, more than 200 , the relevance - the number of program modules, more than 42 , the cost - the complexity of manufacturing 70 person-years. As defined constraints can be selected: the total budget for the production, the minimum acceptable quality of documentation for the product, budget of documentation, availability of all products from the documentation.

Thus may be formulated as a mathematical formulation of the selected method and solved the problem of maximizing the quality of the electronic operational documentation.

\section{Synthesis and structure of expert system and IETM}

Most of the original data of maintenance the product are created the stage of formation operational documentation (OD) and include as part into a support system of the product.

The complexity of created equipment is constantly increasing, there are more documents and operating instructions. Processes of modernization products, documentation development, it becomes more difficult to support.

An effective approach to solving the problem is to automate the process of creating an electronic operational documentation (EOD).

The objectives of creating an automated system EOD are:

- reducing the time and labor intensity of creation and modification;

- rapid change of documentation while maintaining the invariant components and making changes in all structural components of OD using network technologies;

- content in an identical condition in a single database of all language versions of sets OD.

IETM and ES can be considered as a software package for witch creating and maintaining is automated with the using of systemic viewers focused on the given subject area.

Structural information support of IETM and ES must be composed of similar data from the relevant paper operational documents. These data are structured in logical chunks called data modules (MD), consist of text or hypertext media fragments.

Of the full range of the typical tasks of operation and maintenance using IETM with ES should allocate following tasks:

- mathematical formulation;

- analytical study;

- maximizing quality.

Consider the synthesized structure, substantially consisting of two systems - ES and IETM.

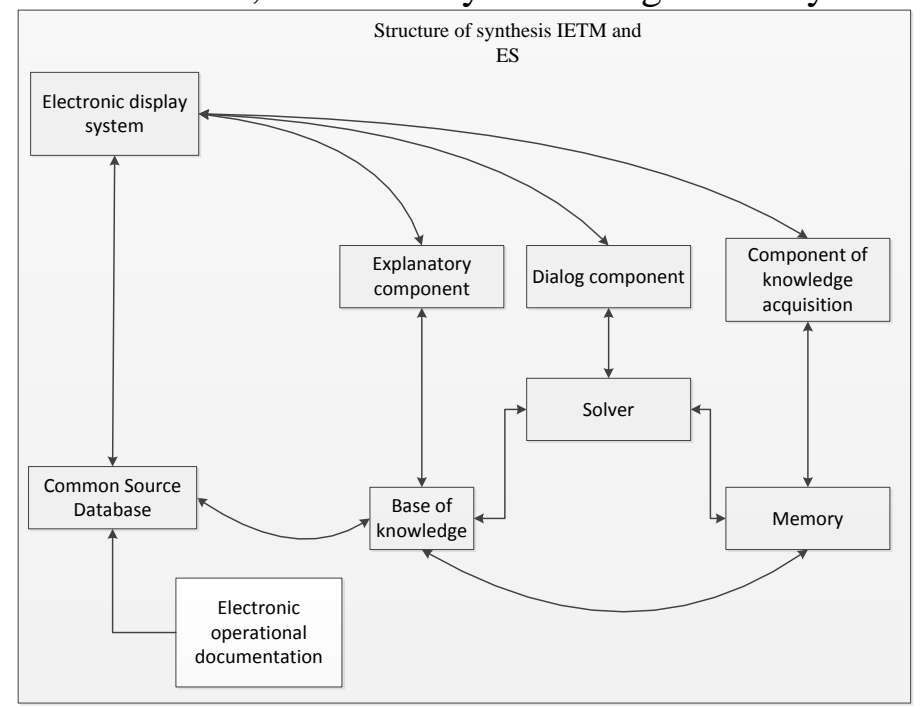


Fig. 1 Structure of synthesis IETM and ES

Figure 1 shows the following components:

The knowledge base contains facts known expert on products.

Explanatory component supports structural and management knowledge. He explains the resulting solution of the system and what knowledge is used in this case.

Dialog component focuses on the communication with the user, as in the solution of problems and in the process of acquiring knowledge and explanation of the results.

The component of knowledge acquisition uses the information on ways representation and modify the knowledge. Automates the process of filling expert systems knowledge, carried out expert users.

The solver uses the information about the process of solving the problem.

Memory contains the accumulated knowledge.

The electronic display system shows the necessary user data.

CSDB contains product documentation.

Information technology development and maintenance of electronic operational documentation is based on the principles of CSDB (Common Source Database) support the product life cycle by means of a shared resource database. Appointment CSDB - Providing basic information to create, refine and modify electronic product documentation. The structure of a common database source data shown in Figure 2.

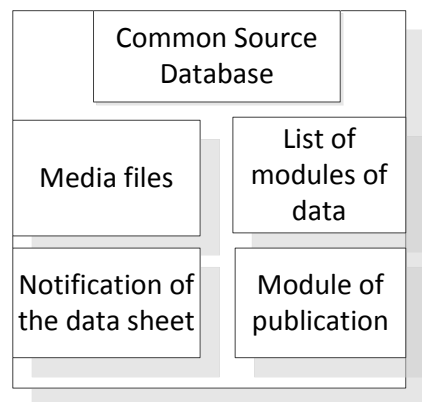

Fig. 2 Common Source Data Base

During the life cycle of products may be modified in the structure refined and varied maintenance that must be recorded in the changes of the content of operational documentation and brought to the attention through a notification about data listing. Changes to the operational documents may be developer and the customer and must be different.

The development of electronic documents of operational documentation is carried out using CSDB, the main objects are data modules, each of which can have more than one version. The original must be a strictly defined [4]. In accordance with Government Standard for the unified system for design(USFD) documentation on each version of MD is an electronic document, but only if approved in a prescribed manner version MD is recognized script. All MD in accordance with USFD is a technical publication (TP) and are aggregated electronic documents. At the same time they are independently of the type of publication (paper, electronic, network, media) consist of copies of the data modules.

Electronic operational documentation (EOD) - Product documentation created during the manufacturing process. Paper operational documentation consists of books, e - from electronic technical publications, consisting of modules of data, just as the book consists of chapters. ETP structure is shown in Figure 3.

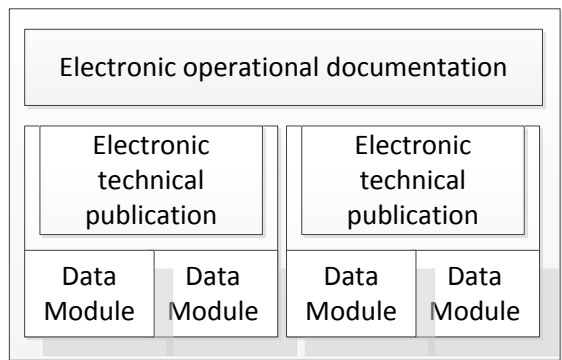

Fig. 3. Electronic operational documentation 
In the electronic operational documentation you can make changes that are made by replacing the approved version of MD in the new version. All changes must be presented in an appropriate change notification specifying the reasons for making changes, and transfer the contents of the changes, time changes to take effect.

Data Module (DM) - represents indivisible unit of a set of specific technical information, united by one theme.

Integration ES and IETM places demands to the compilers of OD in terms of keeping the rules of functioning of ES. Expert describes the problem area totality of facts, rules, and two types of knowledge - declarative and procedural. Declarative knowledge is used to describe the domain of objects and elements of the systems. Procedural knowledge is a set of intellectual abilities of experts in the form of algorithms using knowledge ES.

Database of interactive electronic technical manuals should be constructed of declarative and procedural knowledge, and not only provide the user with information about the items, their description and functioning, but also about support these elements of their operation. The system, based on knowledge, should be able to train users, and from the already acquired knowledge create new ways to solve a particular problem.

The proposed scheme of the system itself should help the user to use the devices and eliminate the long search for information in technical manuals. In this case user does not have independently search a particular node, and in its description search for the needed information. It will be sufficient to interrogate the system for a particular information.

\section{Conclusions}

Identified advantages of using an expert system in interactive electronic technical manuals, such as significant reduction complexity of the design of new and modifying existing products, by reducing the search time previously developed documentation, and by abstracting from the particular automation systems (CAD). It is proposed to develop a new structure based on a synthesis of the two systems and methodology described, which allows to increase the degree of automation of processes analysis documents created in the process of designing and manufacturing application.

\section{References}

[1] Bakayev V. V., Sudov Ye. V., Gomozov V. A. Informatsionnoye Obespecheniye, Podderzhka I Soprovozhdeniye Zhiznennogo Tsikla izdeliya. Moscow: Mashinostroyeniye Press, 2005.

[2] Muromtsev D. I. Introduction to Expert Systems. St. Petersburg: St. Petersburg State University of Information Technologies Press, 2005.

[3] Habarov S. P. Intelligent Information Systems. St. Petersburg: St. Petersburg Forest Technical Academy Press, 2006.

[4] GOST 2.051-2006 ESKD "Electronic documents. General provisions 